\title{
A Nonmonotone Weighting Self-Adaptive Trust Region Algorithm for Unconstrained Nonconvex Optimization
}

\author{
Yunlong Lu, Weiwei Yang, Wenyu Li, Xiaowei Jiang, and Yueting Yang \\ School of Mathematics and Statistics, Beihua University, Jilin 132013, China \\ Correspondence should be addressed to Yueting Yang; yangyueting@163.com
}

Received 14 August 2015; Revised 25 October 2015; Accepted 26 October 2015

Academic Editor: Juan R. Torregrosa

Copyright (C) 2015 Yunlong Lu et al. This is an open access article distributed under the Creative Commons Attribution License, which permits unrestricted use, distribution, and reproduction in any medium, provided the original work is properly cited.

A new trust region method is presented, which combines nonmonotone line search technique, a self-adaptive update rule for the trust region radius, and the weighting technique for the ratio between the actual reduction and the predicted reduction. Under reasonable assumptions, the global convergence of the method is established for unconstrained nonconvex optimization. Numerical results show that the new method is efficient and robust for solving unconstrained optimization problems.

\section{Introduction}

Consider the following unconstrained optimization problem:

$$
\min _{x \in \mathbb{R}^{n}} f(x)
$$

where $f: \mathbb{R}^{n} \rightarrow \mathbb{R}$ is continuously differentiable.

The trust region methods calculate a trial step $d_{k}$ by solving the subproblem at each iteration,

$$
\begin{array}{ll}
\min & q_{k}(d)=f\left(x_{k}\right)+\frac{1}{2} d^{T} B_{k} d+g_{k}^{T} d \\
\text { s.t. } & \|d\| \leq \Delta_{k},
\end{array}
$$

where $g_{k}=\nabla f\left(x_{k}\right)$ and $B_{k}$ is symmetric matrix approximating the Hessian of $f(x)$ at $x_{k}$ and $\Delta_{k}>0$ is a trust region radius at $x_{k}$. Throughout this paper, $\|\cdot\|$ denotes the Euclidean norm on $\mathbb{R}^{n}$. Define the ratio

$$
r_{k}^{O}=\frac{f\left(x_{k}\right)-f\left(x_{k}+d_{k}\right)}{q_{k}(0)-q_{k}\left(d_{k}\right)},
$$

and the numerator and the denominator are called the actual reduction and the predicted reduction, respectively.

The nonmonotone line search technique is firstly proposed by Grippo et al. [1] in line search framework for
Newton's method. At each iteration, the selected function value is taken as

$$
f_{l(k)}=\max _{0 \leq j \leq m(k)} f\left(x_{k-j}\right),
$$

where $m(0)=0,0 \leq m(k) \leq \min \{m(k-1)+1, M\}, M$ is a positive integer. Although many algorithms based on (4) work well in many cases, a "good" function value generated as the iteration process is not selected because of the max function in (4), and the choice of $M$ is sensitive to some numerical tests sometimes. To overcome shortages, Zhang and Hager [2] proposed a new nonmonotone line search technique and they used $C_{k}$ to replace the function in (4), where

$$
C_{k}=\frac{\zeta_{k-1} Q_{k-1} C_{k-1}+f\left(x_{k}\right)}{Q_{k}},
$$

where $Q_{k}=\zeta_{k-1} Q_{k-1}+1, Q_{0}=1$, and $C_{0}=f\left(x_{0}\right), \zeta_{k-1} \in$ $[0,1]$. Numerical tests have shown that the new nonmonotone algorithm is more effective.

Many researchers proposed many trust region methods by considering the ratio $r_{k}$ and the updating trust region radius to solve effectively unconstrained optimization problem. Dai and $\mathrm{Xu}$ [3] proposed the following weighting formula:

$$
\widehat{r}_{k}=\sum_{i=0}^{\min \{k, m\}} \omega_{k i} r_{k-i}^{O},
$$


where $m$ is some positive integer and $\omega_{k i} \in[0,1]$ is the weight of $r_{k-i}$, such that

$$
\sum_{i=0}^{\min \{k, m\}} \omega_{k i}=1
$$

Many self-adaptive adjustment strategies are developed to update the trust region radius, such as [4-14]. In addition, many adaptive nonmonotonic trust region methods have been proposed in literatures [15-21].

In this paper, we propose a new self-adaptive weighting trust region method based on the nonmonotone technique (4) in [2], the weighting technique (5) in [3], and $L$-function in [6].

The rest of the paper is organized as follows. In Section 2, we define $L$-function to introduce a new update rule and a new nonmonotone self-adaptive trust region algorithm is presented. In Section 3, the convergence properties of the proposed algorithm are investigated. In Section 4, numerical results are given. In Section 5 conclusions are summarized.

\section{2. $L$-Function and the New Nonmonotone Self-Adaptive Trust Region Algorithm}

To obtain the new trust region radius update rules, we recall $L$-function $L(t), t \in \mathbb{R}$.

Definition 1 (see [6]). A function $L(t)$ is called an $L$-function if it satisfies the following.

(1) $L(t)$ is nondecreasing in $\left(-\infty, \eta_{2}\right]$ and nonincreasing in $\left(2-\eta_{2},+\infty\right), L(t)=\beta_{2}$, for $t \in\left[\eta_{2}, 2-\eta_{2}\right]$,

(2) $\lim _{t \rightarrow-\infty} L(t)=c_{1}$,

(3) $L(0)=c_{2}$,

(4) $\lim _{t \rightarrow \eta_{2}} L(t)=1$,

(5) $\lim _{t \rightarrow 0^{+}} L(t)=\beta_{1}$,

(6) $L(t)<1$, for $t>\eta_{3}$,

(7) $\lim _{t \rightarrow+\infty} L(t)=\beta_{3}$,

where the constants $\beta_{1}, \beta_{2}, \beta_{3}, \eta_{2}, \eta_{3}, c_{1}$, and $c_{2}$ are positive constants such that

$$
\begin{aligned}
0 & <c_{1}<c_{2}<\beta_{1} \leq \beta_{3}<1<\beta_{2}, \\
\eta_{3} & >2-\eta_{2}, \\
0.5 & \leq \eta_{2}<1 .
\end{aligned}
$$

Now we describe the new nonmonotone self-adaptive trust region algorithm.

\section{Algorithm 2.}

Step 1. Given $x_{0} \in \mathbb{R}^{n}, B_{0} \in \mathbb{R}^{n \times n}, 0<\eta_{2}<1,0<c_{1}<c_{2}<$ $1,0<\beta_{1} \leq \beta_{3}<1 \leq \beta_{2}$, and $\Delta_{0}>0 ; m$ is a given small positive integer; $\varepsilon \geq 0 ; 0 \leq \zeta_{\min } \leq \zeta_{\max }<1, Q_{0}=1$; and set $k:=0$.

Step 2. If $\left\|g_{k}\right\| \leq \varepsilon$, stop.

Step 3. Solve subproblem (2) to get $d_{k}$.

Step 4. Compute

$$
r_{k}=\frac{\text { Ared }_{k}}{\text { Pred }_{k}}=\frac{C_{k}-f\left(x_{k}+d_{k}\right)}{\phi_{k}(0)-\phi_{k}\left(d_{k}\right)}
$$

and $\widehat{r}_{k}$ by (6). If $\widehat{r}_{k} \leq 0$, go to Step 5. Else go to Step 6 .

Step 5. Choose some $L$-function and compute $\Delta_{k+1}=$ $L\left(\widehat{r}_{k}\right) \Delta_{k}$, and then go to Step 3 .

Step 6. Set $x_{k+1}=x_{k}+d_{k}$. Update the trust region radius

$$
\Delta_{k+1}=L\left(\widehat{r}_{k}\right) \Delta_{k} \text {. }
$$

Step 7. Compute $g_{k+1}$ and $B_{k+1}$; choose $\zeta_{k+1} \in\left[\zeta_{\min }, \zeta_{\max }\right]$, set $k:=k+1$, and go to Step 2 .

\section{Convergence of Algorithm 2}

In the section, we consider the convergence properties of Algorithm 2. We give the following assumption.

Assumption 3. (i) The function $f$ is bounded on the level set $S=\left\{x \mid f(x) \leq f\left(x_{0}\right)\right\}$ and twice continuously differentiable. (ii) The sequence $\left\{B_{k}\right\}$ is uniformly bounded in norm; that is, for some constant $M,\left\|B_{k}\right\| \leq M$. (iii) The solution $d_{k}$ of subproblem (2) satisfies

$$
q_{k}(0)-q_{k}\left(d_{k}\right) \geq \sigma\left\|g_{k}\right\| \min \left\{\Delta_{k}, \frac{\left\|g_{k}\right\|}{\left\|B_{k}\right\|}\right\},
$$

where $\sigma \in(0,1]$.

Lemma 4. Suppose that (i) and (ii) in Assumption 3 hold. Then

$$
\left|f\left(x_{k}+d_{k}\right)-q\left(d_{k}\right)\right| \leq \frac{1}{2} M\left\|d_{k}\right\|^{2}+C\left(\left\|d_{k}\right\|\right)\left\|d_{k}\right\|,
$$

where $C\left(\left\|d_{k}\right\|\right)$ arbitrarily decreases with $d_{k}$ decreasing.

Proof. Since, from Taylor theorem, we have

$$
\begin{aligned}
f\left(x_{k}+d_{k}\right)= & f\left(x_{k}\right)+g_{k}^{T} d_{k} \\
& +\int_{0}^{1}\left[\nabla f\left(x_{k}+t d_{k}\right)-g\left(x_{k}\right)\right]^{T} d_{k} d t,
\end{aligned}
$$

it follows from the definition of $q_{k}(d)$ in (2) that

$$
\begin{aligned}
& \left|f\left(x_{k}+d_{k}\right)-q_{k}\left(d_{k}\right)\right| \\
& \quad=\left|\frac{1}{2} d_{k}^{T} B_{k} d_{k}-\int_{0}^{1}\left[\nabla f\left(x_{k}+t d_{k}\right)-g\left(x_{k}\right)\right]^{T} d_{k} d t\right| \\
& \quad \leq \frac{1}{2} M\left\|d_{k}\right\|^{2}+C\left(\left\|d_{k}\right\|\right)\left\|d_{k}\right\|,
\end{aligned}
$$

where $C\left(\left\|d_{k}\right\|\right)$ arbitrarily decreases with $d_{k}$ decreasing. 
Lemma 5. Assume that the sequence $\left\{x_{k}\right\}$ is generated by Algorithm 2. Then the sequence $\left\{x_{k}\right\} \subseteq S$.

Proof. From Lemma 3.1 in [22] and $x_{0} \in S$ in Assumption 3 (i), we have $\left\{x_{k}\right\} \subseteq S$.

The next lemma shows that the loop through Step 3 to Step 5 cannot cycle infinitely and the sequence $\left\{x_{k}\right\}$ is well defined.

Lemma 6. Suppose that Assumption 3 holds. Assume also $g_{k} \neq$ 0 , and there exists a sufficiently small constant $\bar{\Delta}>0$ such that

$$
\Delta_{k} \leq \bar{\Delta}
$$

Then

$$
\Delta_{k+1} \geq \Delta_{k}
$$

holds.

Proof. By Assumption 3 and $g_{k} \neq 0$, there exist $\varepsilon>0$ and a positive index $k_{0}$ such that

$$
\left\|g_{k}\right\| \geq \varepsilon, \quad \forall k \geq k_{0}
$$

Combining (11), we have that, for $k \geq k_{0}$,

$$
\begin{aligned}
q_{k}(0)-q_{k}\left(d_{k}\right) & \geq \sigma\left\|g_{k}\right\| \min \left\{\Delta_{k}, \frac{g_{k}}{\left\|B_{k}\right\|}\right\} \\
& \geq \sigma \varepsilon \min \left\{\Delta_{k}, \frac{\varepsilon}{M}\right\} .
\end{aligned}
$$

Combining (12) and (18), we have

$$
\begin{aligned}
\left|r_{k}^{O}-1\right| & =\left|\frac{f\left(x_{k}+d_{k}\right)-q_{k}\left(d_{k}\right)}{q_{k}(0)-q_{k}\left(d_{k}\right)}\right| \\
& \leq \frac{(1 / 2) M\left\|d_{k}\right\|^{2}+C\left(\left\|d_{k}\right\|\right)\left\|d_{k}\right\|}{\sigma \varepsilon \min \left\{\Delta_{k}, \varepsilon / M\right\}} \\
& \leq \frac{\Delta_{k}\left(M \Delta_{k}+2 C\left(\left\|d_{k}\right\|\right)\right)}{\sigma \varepsilon \min \left\{\Delta_{k}, \varepsilon / M\right\}} .
\end{aligned}
$$

By (15), we can choose sufficiently small $\bar{\Delta}$ such that

$$
\begin{gathered}
\Delta_{k} \leq \bar{\Delta} \leq \frac{\varepsilon}{M}, \\
M \Delta_{k}+2 C\left(\left\|d_{k}\right\|\right) \leq\left(1-\eta_{2}\right) \sigma \varepsilon,
\end{gathered}
$$

and furthermore, for sufficiently large $k \geq k_{0}$,

$$
\left|r_{k}^{O}-1\right| \leq\left(1-\eta_{2}\right)
$$

For the above $k$, we know that

$$
\left|r_{k+j}^{O}-1\right| \leq 1-\eta_{2}, \quad j=1,2, \ldots, m .
$$

From (6) and (22), for sufficiently large $k \geq k_{0}+m$, we know that $\widehat{r}_{k}$ formulate always the form

$$
\begin{aligned}
\left|\widehat{r}_{k}-1\right| & =\left|\sum_{i=0}^{m} \omega_{k i} r_{k-i}^{O}-\sum_{i=0}^{m} \omega_{k i}\right| \leq \sum_{i=0}^{m} \omega_{k i}\left|r_{k-i}^{O}-1\right| \\
& \leq \sum_{i=0}^{m} \omega_{k i}\left(1-\eta_{2}\right) \leq 1-\eta_{2} .
\end{aligned}
$$

From (23), for sufficient large $k$, we have $2-\eta_{2} \geq \widehat{r}_{k} \geq$ $\eta_{2}$. By Algorithm 2 and the definition of $L$-function, we have $\Delta_{k+1} \geq \Delta_{k}$, where $\Delta_{k}$ falls below $\bar{\Delta}$.

We will show the global convergence of Algorithm 2.

Theorem 7. Suppose that Assumption 3 holds. Let the sequence $\left\{x_{k}\right\}$ be generated by Algorithm 2. Then

$$
\liminf _{k \rightarrow \infty}\left\|g_{k}\right\|=0 .
$$

Proof. For the purpose of deriving a contradiction, suppose that there exists a positive constant $\delta>0$ such that

$$
\left\|g_{k}\right\| \geq \delta>0 .
$$

For convenience, we denote one index set as follows:

$$
J=\left\{k \mid \widehat{r}_{k} \geq \eta_{2}\right\} .
$$

First, assume that the set $J$ has infinite elements. That is, for any $k \in J, \widehat{r}_{k} \geq \eta_{2}$ holds. For any $k \in J$, using Algorithm 2 and (12), we have that

$$
\begin{aligned}
C_{k}-f\left(x_{k+1}\right) & \geq \eta_{2}\left[q_{k}(0)-q_{k}\left(d_{k}\right)\right] \\
& \geq \sigma \eta_{2}\left\|g_{k}\right\| \min \left\{\Delta_{k}, \frac{\left\|g_{k}\right\|}{\left\|B_{k}\right\|}\right\} \\
& \geq \sigma \eta_{2} \delta \min \left\{\Delta_{k}, \frac{\delta}{M}\right\} .
\end{aligned}
$$

Thus, from (27),

$$
f\left(x_{k+1}\right) \leq C_{k}-\sigma \eta_{2} \delta \min \left\{\Delta_{k}, \frac{\delta}{M}\right\} .
$$

From (5) and (28), we have that

$$
\begin{aligned}
C_{k+1} & =\frac{\zeta_{k} Q_{k} C_{k}+f\left(x_{k+1}\right)}{Q_{k+1}} \\
& \leq \frac{\zeta_{k} Q_{k} C_{k}+C_{k}-\sigma \eta_{2} \delta \min \left\{\Delta_{k}, \delta / M\right\}}{Q_{k+1}} \\
& =C_{k}-\frac{\sigma \eta_{2} \delta \min \left\{\Delta_{k}, \delta / M\right\}}{Q_{k+1}} .
\end{aligned}
$$

From Lemma 3.1 in [22] and Assumption 3 (i), we know the sequence $\left\{C_{k}\right\}$ is nonincreasing and convergent. Then

$$
\lim _{k \rightarrow \infty} \Delta_{k}=0
$$


which contradicts (16). Next, we assume that the set $J$ has finite elements. Then, for sufficient large $k$, we have that $\widehat{r}_{k}<$ $\eta_{2}$. From the definition of $L$-function and Steps 5 and 6 in Algorithm 2, we have that the trust region $\Delta_{k}$ is decreasing as the iteration process. Furthermore, the limit

$$
\lim _{k \rightarrow \infty} \Delta_{k}=0
$$

holds, which gives a contradiction to (16). The proof is completed.

\section{Numerical Experiments}

In this section, we present preliminary numerical results to illustrate the performance of Algorithm 2, denoted by NTRW. In Algorithm 2, we choose

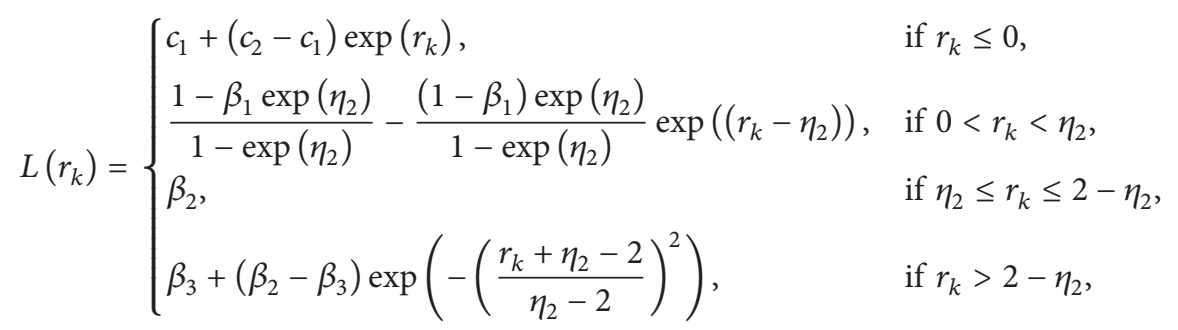

where $\beta_{1}=0.5, \beta_{2}=2, \beta_{3}=0.7, c_{1}=0.12, c_{2}=$ 0.14 , and $\eta_{2}=0.75$. In the framework of Algorithm 2, we compare NTRW with the following algorithms: the basic trust region method, denoted by BTR; the basic trust region method with Grippo's nonmonotone technique, denoted by NTR1, $m=3$; the basic trust region method with Hager's nonmonotone technique, denoted by NTR2, $\zeta=0.5$. All tests are implemented by using Matlab R2008a on a PC with CPU 2.40 GHz and 2.00 GB RAM. The test problem collections for unconstrained minimization in Table 1 are taken from Moré et al. in [23], the CUTEr collection [24, 25].

In all algorithms in this paper, the matrix $B_{k}$ is updated by BFGS formula $[26,27]$. The trial step $d_{k}$, for smallscale problems, is computed by trust $\mathrm{m}$ file in Optimization Toolbox of Matlab, for middle-scale and large-scale problems, and is computed by CG-Steihaug algorithm in [26]. The iteration is terminated by the following condition:

$$
\left\|g_{k}\right\| \leq \varepsilon
$$

where $\varepsilon=10^{-5}$. In Tables 1, 2, 3, and 4, we give the dimension (Dim) of each test problem (P), the number iter of iterations, the number $n f$ of function evaluations, and the CPU (cpu) time for solving the test problem.

In Table 2, we compare 43 small-scale problems for the four algorithms, and the results are concluded as follows:

(i) 19 problems where NTRW was superior to BTR,

(ii) 11 problems where BTR was superior to NTRW,

(iii) 13 problems where NTR2 was superior to NTR1,

(iv) 5 problems where NTR1 was superior to NTR2,

(v) 25 problems where NTRW was superior to NTR2,

(vi) 10 problems where NTR2 was superior to NTRW.

For problems 12, 18, 24, 30, and 36 especially, the iterations of four algorithms are similar while $n f$ of NTRW are much less than the others. It means that the number of subproblem evaluations of NTRW is much less than the others. Therefore, our self-adaptive technique is efficient. For problems 10, 20, 27,32 , and 38 , NTRW is superior to the others clearly. And cpu of NTRW is less than the others. So the performance of our algorithm is better than the others.

In Table 3, we compare 25 middle-scale problems of the four algorithms. There are 12 problems that show NTRW is much superior than the others, 7 problems that show the performance of the four algorithms is similar, and only 4 problems that show NTRW is bad.

In Table 4, we compare 10 large-scale problems of the four algorithms. There are 5 problems that show NTRW is much superior than the others, 4 problems that show the performance of the four algorithms is similar, and only 1 problem that shows NTRW is bad. Note that, for problems 33 and 35, the iteration of our algorithm is similar to NTR2, while the CPU time is much more than NTR2. Exponential function called in Matlab environment maybe consume more time, which is contained in $L$-function in (32).

Further result is shown in Figures 1 and 2, which is characterized by means of performance profile proposed in [28]. The performance ratio $q(\tau)$ is the probability for solver $s$ for the test problems, where a log-scale ratio is not greater than the factor $\tau$. More details are founded in [28]. As we can see from Figures 1 and 2, NTRW is obviously superior than NTR1 and NTR2 in the number of iterations and function evaluations. NTRW is superior than the other three algorithms in the number of function evaluations.

\section{Conclusion}

This paper presents a nonmonotone weighting self-adaptive trust region algorithm for unconstrained nonconvex optimization. The new algorithm is very simple and easily implemented. The convergence properties of the method 
TABLe 1: Test problems.

\begin{tabular}{|c|c|}
\hline Number & Problem name \\
\hline 1 & Helical valley function \\
\hline 2 & Biggs EXP6 function \\
\hline 3 & Gaussian function \\
\hline 4 & Box function \\
\hline 5 & Variable dimension function \\
\hline 6 & Watson function \\
\hline 7 & Penalty function I \\
\hline 8 & Penalty function II \\
\hline 9 & Brown badly scaled function \\
\hline 10 & Brown and Dennis function \\
\hline 11 & Gulf function \\
\hline 12 & Extended Rosenbrock function \\
\hline 13 & Beale function \\
\hline 14 & Wood function \\
\hline 15 & Chebyquad function \\
\hline 16 & Boundary value function \\
\hline 17 & Separable cubic function \\
\hline 18 & Powell singular function \\
\hline 19 & Linear function, full rank \\
\hline 20 & Linear function, rank 1 \\
\hline 21 & FLETCHCR function \\
\hline 22 & BDQRTIC function \\
\hline 23 & TRIDIA function \\
\hline 24 & ARGLINB function \\
\hline 25 & ARWHEAD function \\
\hline 26 & NONDIA function \\
\hline 27 & NONDQUAR function \\
\hline 28 & Generalized Rosenbrock function \\
\hline 29 & Broyden tridiagonal function \\
\hline 30 & Allgower function \\
\hline 31 & EG2 function \\
\hline 32 & CURLY20 function \\
\hline 33 & LIARWHD function \\
\hline 34 & POWER function \\
\hline 35 & ENGVAL1 function \\
\hline 36 & ARGLINC function \\
\hline 37 & NONSCOMP function \\
\hline 38 & VARDIM function \\
\hline 39 & QUARTC function \\
\hline 40 & Extended DENSCHNB function \\
\hline 41 & Extended DENSCHNF function \\
\hline 42 & DIXON3DQ function \\
\hline 43 & BiGGSB1 function \\
\hline 44 & Nearly separable function \\
\hline 45 & Schittkowski function 302 \\
\hline 46 & Discrete integral equation function \\
\hline 47 & DQDRTIC function \\
\hline 48 & EDENSCH function \\
\hline 49 & Bdexp function \\
\hline 50 & COSINE function \\
\hline 51 & HIMMELBG function \\
\hline
\end{tabular}

TABLE 2: Numerical comparisons for some small-scale test problems.

\begin{tabular}{|c|c|c|c|c|}
\hline P/Dim & $\begin{array}{c}\text { BTR } \\
\text { iter/nf }\end{array}$ & $\begin{array}{l}\text { NTR1 } \\
\text { iter/nf }\end{array}$ & $\begin{array}{l}\text { NTR2 } \\
\text { iter/nf }\end{array}$ & $\begin{array}{c}\text { NTRW } \\
\text { iter/nf }\end{array}$ \\
\hline $1 / 3$ & $32 / 43$ & $31 / 51$ & $30 / 51$ & $38 / 45$ \\
\hline $2 / 6$ & $38 / 45$ & $37 / 42$ & $36 / 41$ & $40 / 41$ \\
\hline $3 / 3$ & $3 / 4$ & $3 / 4$ & $3 / 4$ & $3 / 4$ \\
\hline $4 / 3$ & $33 / 47$ & $33 / 46$ & $33 / 46$ & $38 / 41$ \\
\hline $5 / 5$ & $21 / 30$ & $11 / 28$ & $11 / 28$ & $14 / 19$ \\
\hline $6 / 5$ & $23 / 31$ & $23 / 32$ & $24 / 35$ & $24 / 27$ \\
\hline $7 / 5$ & $188 / 240$ & $19 / 36$ & $19 / 36$ & $65 / 76$ \\
\hline $8 / 5$ & $18 / 26$ & $18 / 26$ & $18 / 26$ & $8 / 11$ \\
\hline $9 / 2$ & $69 / 124$ & $* *$ & $* *$ & $105 / 117$ \\
\hline $10 / 4$ & $72 / 92$ & $219 / 349$ & $184 / 261$ & $44 / 57$ \\
\hline $11 / 3$ & $9 / 10$ & $9 / 10$ & $9 / 10$ & $9 / 10$ \\
\hline $12 / 6$ & $41 / 62$ & $44 / 76$ & $44 / 72$ & $51 / 58$ \\
\hline $13 / 2$ & $12 / 15$ & $17 / 20$ & $17 / 20$ & $15 / 17$ \\
\hline $14 / 4$ & $34 / 50$ & $140 / 254 /$ & $140 / 188$ & $104 / 125$ \\
\hline $15 / 5$ & $8 / 10$ & $8 / 10$ & $8 / 10$ & $9 / 11$ \\
\hline $16 / 10$ & $23 / 27$ & $23 / 34$ & $23 / 28$ & $20 / 22$ \\
\hline $17 / 10$ & $8 / 9$ & $8 / 9$ & $8 / 9$ & $8 / 9$ \\
\hline $18 / 4$ & $32 / 44$ & $26 / 36$ & $33 / 43$ & $27 / 31$ \\
\hline $19 / 10$ & $3 / 4$ & $3 / 4$ & $3 / 4$ & $3 / 4$ \\
\hline $20 / 10$ & $7 / 20$ & $14 / 48$ & $14 / 48$ & $3 / 8$ \\
\hline $21 / 10$ & $38 / 58$ & $43 / 94$ & $44 / 98$ & $49 / 62$ \\
\hline $22 / 10$ & $35 / 48$ & $35 / 79$ & $35 / 80$ & $39 / 50$ \\
\hline $23 / 10$ & $33 / 44$ & $32 / 64$ & $32 / 57$ & $52 / 64$ \\
\hline $24 / 10$ & $* *$ & $* *$ & $* *$ & $* *$ \\
\hline $25 / 10$ & $9 / 13$ & $9 / 14$ & $9 / 14$ & $11 / 15$ \\
\hline $26 / 10$ & $43 / 80$ & $24 / 58$ & $24 / 58$ & $76 / 93$ \\
\hline $27 / 10$ & 98/102 & $98 / 105$ & $98 / 105$ & $85 / 93$ \\
\hline $28 / 20$ & $110 / 150$ & $107 / 203$ & $102 / 182$ & $119 / 147$ \\
\hline $29 / 20$ & $30 / 56$ & $30 / 82$ & $30 / 82$ & $57 / 72$ \\
\hline $30 / 20$ & $65 / 93$ & $69 / 138$ & $75 / 127$ & $64 / 86$ \\
\hline $31 / 20$ & $22 / 24$ & $22 / 24$ & $24 / 26$ & $23 / 25$ \\
\hline $32 / 20$ & $* *$ & $64 / 145$ & $63 / 131$ & $65 / 81$ \\
\hline $33 / 20$ & $30 / 58$ & $30 / 72$ & $30 / 72$ & $40 / 51$ \\
\hline $34 / 20$ & $74 / 107$ & $77 / 157$ & $77 / 145$ & $92 / 115$ \\
\hline $35 / 20$ & $28 / 55$ & $26 / 77$ & $25 / 70$ & $42 / 50$ \\
\hline $36 / 30$ & $* *$ & $* *$ & $* *$ & $* *$ \\
\hline $37 / 30$ & $346 / 475$ & $388 / 580$ & $334 / 455$ & $258 / 321$ \\
\hline $38 / 30$ & $* *$ & $* *$ & $* *$ & $60 / 70$ \\
\hline $39 / 30$ & $17 / 18$ & $17 / 18$ & $17 / 18$ & $19 / 20$ \\
\hline $40 / 30$ & $8 / 9$ & $8 / 9$ & $8 / 9$ & $8 / 9$ \\
\hline $41 / 30$ & $37 / 76$ & $38 / 100$ & $38 / 100$ & $47 / 57$ \\
\hline $42 / 30$ & $37 / 64$ & $34 / 68$ & $36 / 69$ & $53 / 63$ \\
\hline $43 / 30$ & $36 / 59$ & $37 / 73$ & $38 / 79$ & $52 / 62$ \\
\hline
\end{tabular}

** means that the algorithm reaches 500 iterations. 
TABLE 3: Numerical comparisons for some middle-scale test problems.

\begin{tabular}{|c|c|c|c|c|}
\hline P/Dim & $\begin{array}{c}\text { BTR } \\
\text { iter/nf/cpu }\end{array}$ & $\begin{array}{c}\text { NTR1 } \\
\text { iter/nf/cpu }\end{array}$ & $\begin{array}{c}\text { NTR2 } \\
\text { iter/nf/cpu }\end{array}$ & $\begin{array}{c}\text { NTRW } \\
\text { iter/nf/cpu }\end{array}$ \\
\hline $5 / 500$ & $45 / 114 / 0.64$ & $160 / 534 / 1.47$ & $369 / 1192 / 3.01$ & $73 / 92 / 2.85$ \\
\hline $7 / 500$ & $113 / 228 / 4.35$ & $123 / 361 / 2.79$ & $259 / 700 / 5.57$ & $86 / 99 / 3.99$ \\
\hline $16 / 500$ & $6 / 11 / 0.33$ & $16 / 36 / 0.78$ & $9 / 23 / 0.44$ & $7 / 8 / 0.19$ \\
\hline $17 / 500$ & $8 / 10 / 0.64$ & $8 / 10 / 0.69$ & $8 / 10 / 0.75$ & $9 / 11 / 0.78$ \\
\hline $22 / 500$ & $* *$ & $227 / 500 / 15.60$ & $251 / 450 / 16.05$ & $* *$ \\
\hline $23 / 500$ & $4407 / 7001 / 211.49$ & $4205 / 11668 / 253.84$ & $4179 / 8214 / 213.60$ & $* *$ \\
\hline $25 / 500$ & $16 / 31 / 0.56$ & $11 / 28 / 0.42$ & $11 / 28 / 0.36$ & $28 / 35 / 1.09$ \\
\hline $26 / 500$ & $21 / 40 / 0.70$ & $308 / 552 / 2.26$ & $196 / 310 / 1.84$ & $10 / 15 / 0.37$ \\
\hline $29 / 500$ & $149 / 236 / 7.43$ & $153 / 471 / 9.64$ & $153 / 358 / 7.63$ & $109 / 142 / 4.73$ \\
\hline $31 / 500$ & $13 / 24 / 0.55$ & $15 / 30 / 0.62$ & $20 / 41 / 0.75$ & $50 / 55 / 2.04$ \\
\hline $33 / 500$ & $26 / 38 / 1.11$ & $24 / 56 / 0.86$ & $31 / 80 / 1.05$ & $33 / 43 / 1.37$ \\
\hline $35 / 500$ & $42 / 96 / 2.53$ & $43 / 144 / 2.84$ & $37 / 134 / 2.67$ & $56 / 66 / 3.32$ \\
\hline $37 / 500$ & $* *$ & $66 / 224 / 4.60$ & $71 / 227 / 4.74$ & $54 / 65 / 3.10$ \\
\hline $38 / 500$ & $62 / 135 / 1.31$ & $202 / 666 / 2.64$ & $354 / 1182 / 3.14$ & $73 / 92 / 3.03$ \\
\hline $39 / 500$ & $14 / 18 / 0.83$ & $14 / 18 / 0.75$ & $14 / 18 / 0.70$ & $12 / 14 / 0.58$ \\
\hline $40 / 500$ & $8 / 13 / 0.48$ & $11 / 16 / 0.56$ & $11 / 16 / 0.70$ & $7 / 9 / 0.31$ \\
\hline $41 / 500$ & $* *$ & $* *$ & $37 / 183 / 0.39$ & $27 / 33 / 1.20$ \\
\hline $44 / 500$ & $* *$ & $116 / 280 / 8.85$ & $2686 / 3879 / 248.02$ & $50 / 62 / 4.62$ \\
\hline $45 / 500$ & $49 / 108 / 0.90$ & $134 / 457 / 1.29$ & 257/852/1.98 & $80 / 96 / 2.28$ \\
\hline $46 / 500$ & $76 / 208 / 59.83$ & $140 / 486 / 85.19$ & $* *$ & $* *$ \\
\hline $47 / 500$ & $67 / 116 / 3.85$ & $* *$ & $84 / 224 / 3.40$ & $137 / 162 / 7.36$ \\
\hline $48 / 500$ & $35 / 80 / 1.67$ & $33 / 131 / 2.15$ & $33 / 112 / 1.83$ & $* *$ \\
\hline $49 / 500$ & $18 / 19 / 1.00$ & $18 / 19 / 1.05$ & $18 / 19 / 1.00$ & $24 / 25 / 1.48$ \\
\hline $50 / 500$ & $90 / 154 / 5.80$ & $42 / 127 / 3.56$ & $88 / 204 / 6.33$ & $63 / 71 / 3.74$ \\
\hline $51 / 500$ & $7 / 8 / 0.45$ & 7/8/0.39 & $7 / 8 / 0.33$ & $28 / 29 / 1.50$ \\
\hline
\end{tabular}

** means that the algorithm reaches 5000 iterations.

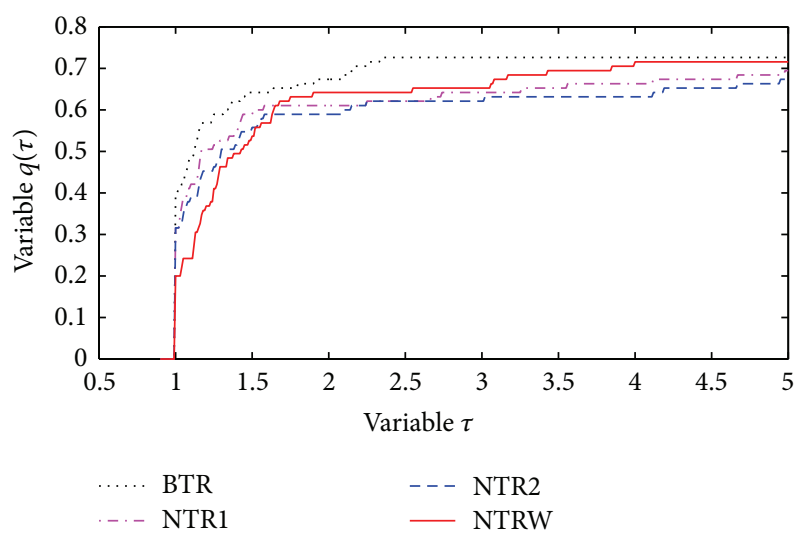

FIGURE 1: Performance profile comparing the number of iterations.

are established under reasonable assumptions. Numerical experiments show that the new algorithm is quite robust and effective, and the numerical performance is comparable to or better than that of other trust region algorithms in the same frame.

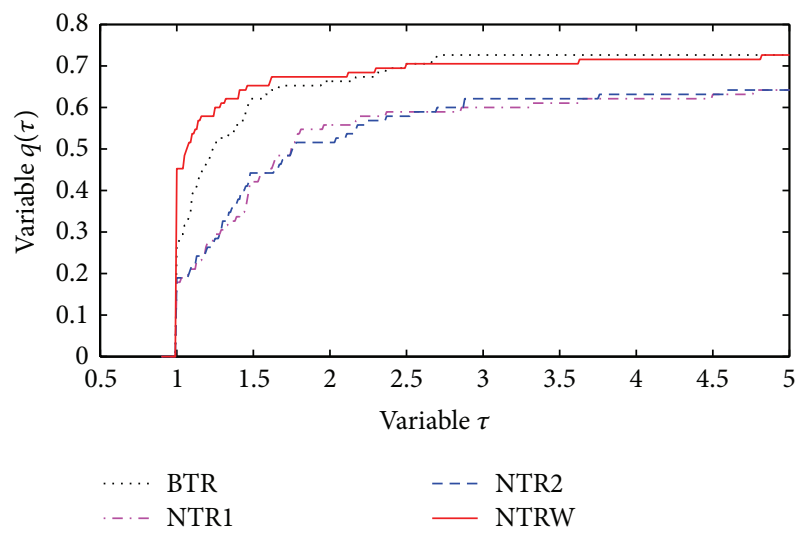

Figure 2: Performance profile comparing the number of function evaluations.

\section{Conflict of Interests}

The authors declare that they have no conflict of interests. 
TABLE 4: Numerical comparisons for some large-scale test problems.

\begin{tabular}{|c|c|c|c|c|}
\hline P/Dim & $\begin{array}{c}\text { BTR } \\
\text { iter/nf/cpu }\end{array}$ & $\begin{array}{c}\text { NTR1 } \\
\text { iter/nf/cpu }\end{array}$ & $\begin{array}{c}\text { NTR2 } \\
\text { iter/nf/cpu }\end{array}$ & $\begin{array}{c}\text { NTRW } \\
\text { iter/nf/cpu }\end{array}$ \\
\hline $26 / 1000$ & $37 / 54 / 3.74$ & $603 / 1072 / 9.20$ & $220 / 358 / 4.44$ & $221 / 260 / 39.12$ \\
\hline $26 / 3000$ & $56 / 75 / 44.42$ & $1787 / 3148 / 174.64$ & $642 / 929 / 75.06$ & $491 / 582 / 431.90$ \\
\hline $29 / 1000$ & $10 / 12 / 3.60$ & $10 / 12 / 3.04$ & $10 / 12 / 3.16$ & $8 / 10 / 2.26$ \\
\hline $29 / 3000$ & $10 / 13 / 14.18$ & $10 / 13 / 14.35$ & $10 / 13 / 14.07$ & 7/9/9.76 \\
\hline $33 / 1000$ & $20 / 35 / 1.99$ & $27 / 65 / 2.91$ & $99 / 199 / 4.66$ & $24 / 30 / 2.87$ \\
\hline $33 / 3000$ & $56 / 115 / 46.00$ & $36 / 86 / 16.34$ & $26 / 59 / 12.38$ & $80 / 95 / 83.33$ \\
\hline $33 / 5000$ & $25 / 40 / 45.81$ & $29 / 70 / 27.12$ & $39 / 87 / 45.13$ & $38 / 50 / 81.99$ \\
\hline $37 / 1000$ & $55 / 186 / 9.47$ & $55 / 197 / 9.68$ & $55 / 186 / 9.34$ & $57 / 69 / 7.80$ \\
\hline $37 / 3000$ & - & - & - & $48 / 57 / 35.81$ \\
\hline $37 / 5000$ & - & - & - & $102 / 123 / 186.96$ \\
\hline $38 / 1000$ & $95 / 202 / 7.83$ & $1058 / 3335 / 21.27$ & $* *$ & 25/47/1.76 \\
\hline $38 / 3000$ & - & - & - & $19 / 45 / 9.67$ \\
\hline $39 / 1000$ & $18 / 22 / 3.58$ & $16 / 20 / 2.99$ & $16 / 20 / 4.16$ & $18 / 20 / 2.67$ \\
\hline $39 / 3000$ & $18 / 23 / 14.00$ & $17 / 22 / 13.63$ & $17 / 22 / 12.13$ & $28 / 31 / 21.29$ \\
\hline $39 / 5000$ & $18 / 23 / 33.38$ & $16 / 22 / 29.99$ & $16 / 22 / 28.34$ & $28 / 31 / 52.21$ \\
\hline $40 / 1000$ & $7 / 13 / 2.45$ & $8 / 18 / 2.45$ & $8 / 18 / 2.54$ & $8 / 11 / 2.65$ \\
\hline $40 / 3000$ & $7 / 15 / 11.04$ & $9 / 21 / 12.18$ & $9 / 21 / 12.64$ & $9 / 12 / 13.85$ \\
\hline $40 / 5000$ & $7 / 14 / 22.92$ & $796 / 2482 / 436.10$ & $15 / 29 / 27.44$ & $10 / 13 / 30.00$ \\
\hline $41 / 1000$ & $* *$ & $* *$ & $39 / 196 / 2.39$ & $29 / 38 / 9.73$ \\
\hline $41 / 3000$ & - & - & $47 / 236 / 12.35$ & $10 / 15 / 9.07$ \\
\hline $41 / 5000$ & - & - & $49 / 246 / 34.24$ & $22 / 30 / 41.85$ \\
\hline $44 / 1000$ & $120 / 327 / 32.46$ & - & $120 / 327 / 22.86$ & $91 / 113 / 34.32$ \\
\hline $44 / 3000$ & - & - & $112 / 472 / 66.40$ & $80 / 98 / 111.91$ \\
\hline $44 / 5000$ & - & - & - & $79 / 95 / 224.81$ \\
\hline $50 / 1000$ & $44 / 89 / 17.55$ & $48 / 143 / 22.74$ & $61 / 158 / 26.38$ & $65 / 73 / 26.05$ \\
\hline $50 / 3000$ & $36 / 77 / 36.08$ & - & - & $78 / 87 / 57.06$ \\
\hline $50 / 5000$ & $161 / 250 / 341.79$ & - & - & $58 / 66 / 108.62$ \\
\hline
\end{tabular}

- means that the algorithm does not end in 30 minutes; $* *$ means that the algorithm reaches 5000 iterations.

\section{Acknowledgment}

This research is partly supported by Chinese NSF under Grant no. 11171003.

\section{References}

[1] L. Grippo, F. Lampariello, and S. Lucidi, "A nonmonotone line search technique for Newton's method," SIAM Journal on Numerical Analysis, vol. 23, no. 4, pp. 707-716, 1986.

[2] H. C. Zhang and W. W. Hager, "A nonmonotone line search technique and its application to unconstrained optimization," SIAM Journal on Optimization, vol. 14, no. 4, pp. 1043-1056, 2004.

[3] Y. H. Dai and D. C. Xu, "A new family of trust region algorithms for unconstrained optimization," Journal of Computational Mathematics, vol. 21, pp. 221-228, 2003.

[4] A. R. Conn, N. I. M. Gould, and P. L. Toint, Trust Region Methods, vol. 1 of MPS/SIAM Series on Optimization, SIAM, Philadelphia, Pa, USA, 2000.

[5] J. H. Fu, W. Y. Sun, and R. J. De Sampaio, "An adaptive approach of conic trust region method for unconstrained optimization problems," Journal of Applied Mathematics \& Computing, vol. 19, no. 1-2, pp. 165-177, 2005.

[6] Y. L. Lu, W. Y. Li, M. Y. Cao, and Y. T. Yang, "A novel self-adaptive trust region algorithm for unconstrained optimization," Journal of Applied Mathematics, vol. 2014, Article ID 610612, 8 pages, 2014.

[7] N. I. M. Gould, D. Orban, A. Sartenaer, and P. L. Toint, "Sensitivity of trust-region algorithms to their parameters," 4OR, vol. 3, no. 3, pp. 227-241, 2005.

[8] L. Hei, "A self-adaptive trust region algorithm," Journal of Computational Mathematics, vol. 21, no. 2, pp. 229-236, 2003.

[9] A. Sartenaer, "Automatic determination of an initial trust region in nonlinear programming," SIAM Journal on Scientific Computing, vol. 18, no. 6, pp. 1788-1803, 1997.

[10] Z.-J. Shi and J.-H. Guo, "A new trust region method for unconstrained optimization," Journal of Computational and Applied Mathematics, vol. 213, no. 2, pp. 509-520, 2008.

[11] Z. Y. Sang and Q. Y. Sun, "A self-adaptive trust region method with line search based on a simple subproblem model," Journal of Computational and Applied Mathematics, vol. 232, no. 2, pp. 514-522, 2009.

[12] J. M. B. Walmag and E. J. M. Delhez, "A note on trust-region radius update," SIAM Journal on Optimization, vol. 16, no. 2, pp. 548-562, 2005. 
[13] Z. S. Yu and Q. Li, "A self-adaptive trust region method for the extended linear complementarity problems," Applications of Mathematics, vol. 54, no. 1, pp. 53-65, 2009.

[14] X. Zhang, J. Zhang, and L. Liao, "An adaptive trust region method and its convergence," Science in China. Series A. Mathematics, vol. 45, no. 5, pp. 620-631, 2002.

[15] M. Ahookhosh and K. Amini, "A nonmonotone trust region method with adaptive radius for unconstrained optimization problems," Computers \& Mathematics with Applications, vol. 60, no. 3, pp. 411-422, 2010.

[16] Z. C. Cui and B. Y. Wu, "A new modified nonmonotone adaptive trust region method for unconstrained optimization," Computational Optimization and Applications, vol. 53, no. 3, pp. 795-806, 2012.

[17] J. H. Fu and W. Y. Sun, "Nonmonotone adaptive trust-region method for unconstrained optimization problems," Applied Mathematics and Computation, vol. 163, no. 1, pp. 489-504, 2005.

[18] Z. J. Shi and S. Q. Wang, "Nonmonotone adaptive trust region method," European Journal of Operational Research, vol. 208, no. 1, pp. 28-36, 2011.

[19] Z. Sang and Q. Sun, "A new non-monotone self-adaptive trust region method for unconstrained optimization," Journal of Applied Mathematics and Computing, vol. 35, no. 1-2, pp. 53-62, 2011.

[20] J.-L. Zhang and X.-S. Zhang, "A nonmonotone adaptive trust region method and its convergence," Computers \& Mathematics with Applications, vol. 45, no. 10-11, pp. 1469-1477, 2003.

[21] J. Zhang, K. C. Zhang, and S. J. Qu, "A nonmonotone adaptive trust region method for unconstrained optimization based on conic model," Applied Mathematics and Computation, vol. 217, no. 8, pp. 4265-4273, 2010.

[22] J. T. Mo, C. Y. Liu, and S. C. Yan, "A nonmonotone trust region method based on nonincreasing technique of weighted average of the successive function values," Journal of Computational and Applied Mathematics, vol. 209, no. 1, pp. 97-108, 2007.

[23] J. J. Moré, B. S. Garbow, and K. E. Hillstrom, "Testing unconstrained optimization software," ACM Transactions on Mathematical Software, vol. 7, no. 1, pp. 17-41, 1981.

[24] N. I. M. Gould, D. Orban, and P. L. Toint, "CUTEr and SifDec: a constrained and unconstrained testing environment, revisited," ACM Transactions on Mathematical Software, vol. 29, no. 4, pp. 373-394, 2003.

[25] N. Andrei, "An unconstrained optimization test functions collection," Advanced Modeling and Optimization, vol. 10, no. 1, pp. 147-161, 2008.

[26] J. Nocedal and S. T. Wright, Numerical Optimization, Springer, Berlin, Germany, 2000.

[27] W. Sun and Y. Yuan, Optimization Theory and Methods. Nonlinear Programming, Springer, New York, NY, USA, 2006.

[28] E. D. Dolan and J. J. Moré, "Benchmarking optimization software with performance profiles," Mathematical Programming, vol. 91, no. 2, pp. 201-213, 2002. 


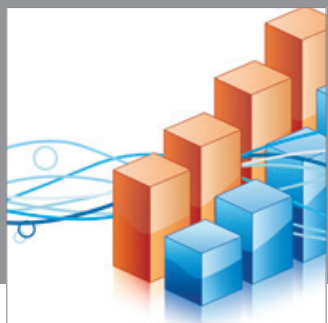

Advances in

Operations Research

mansans

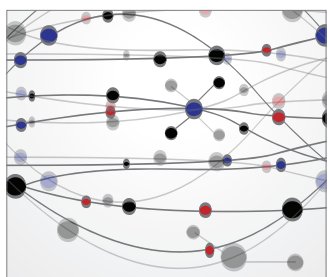

The Scientific World Journal
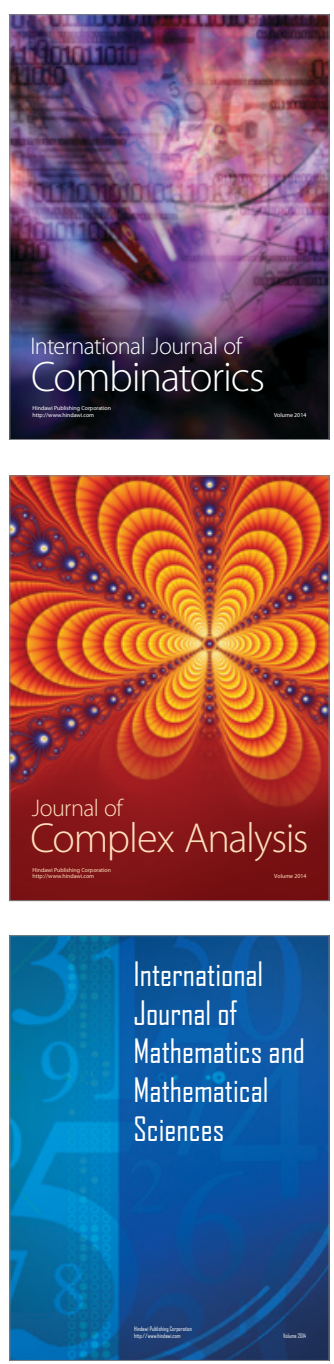
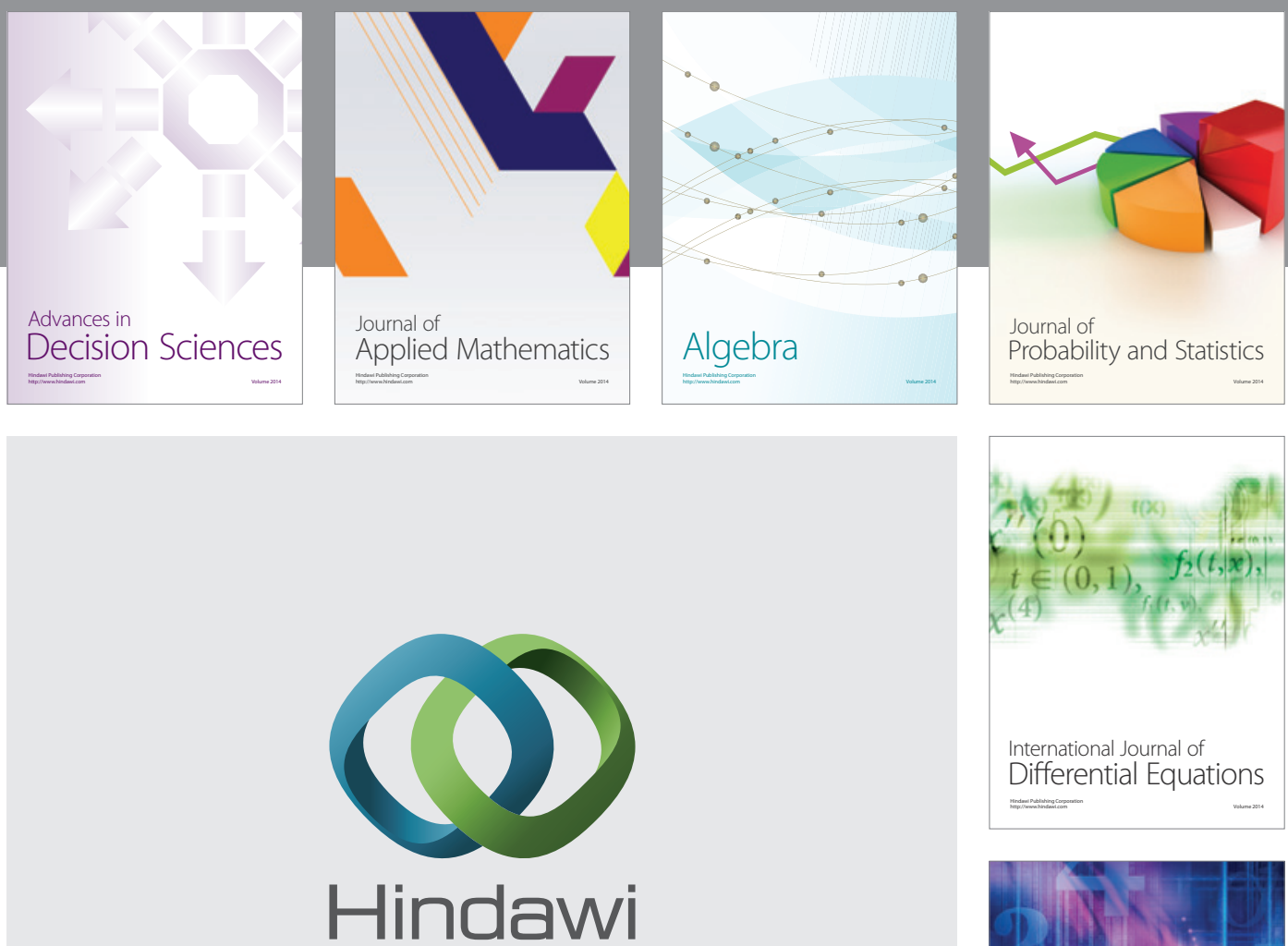

Submit your manuscripts at http://www.hindawi.com
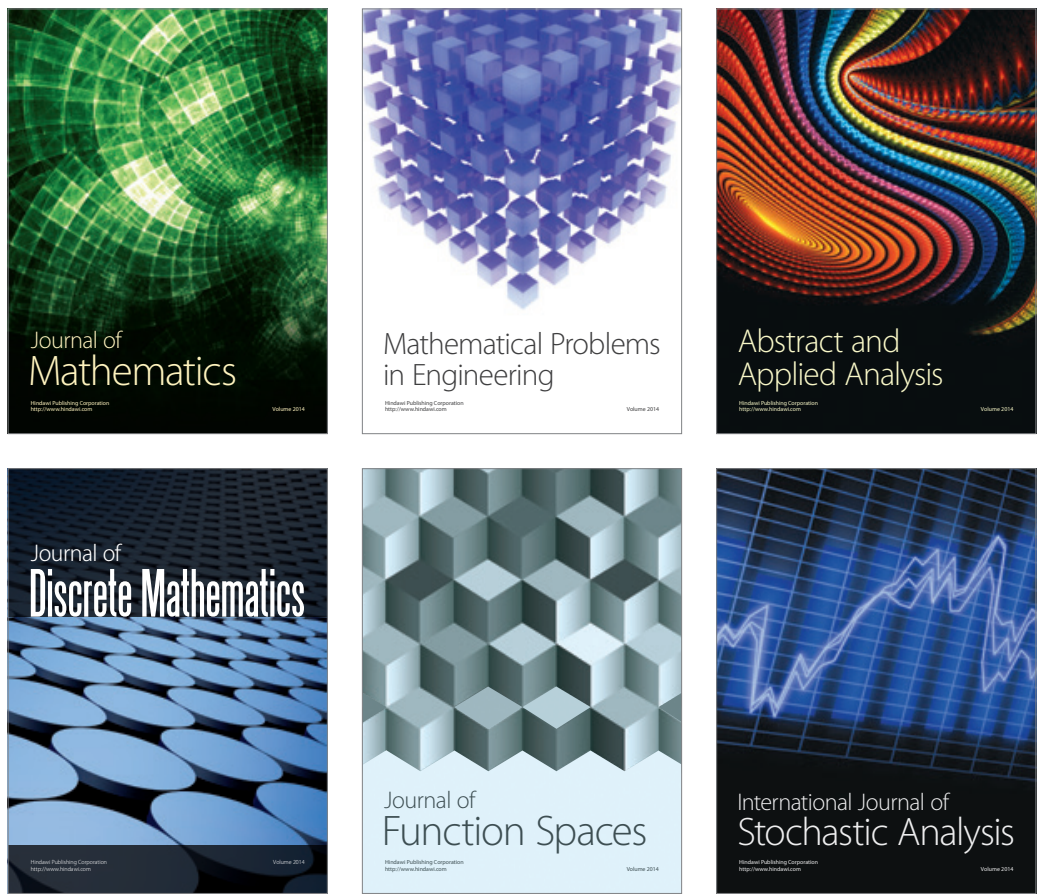

Journal of

Function Spaces

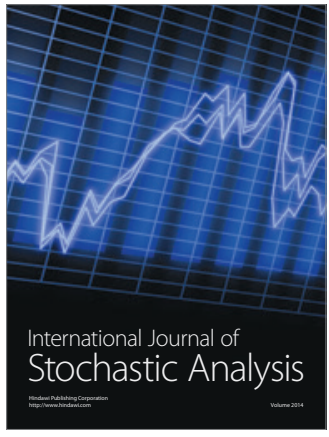

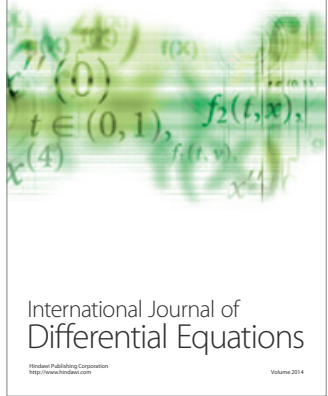
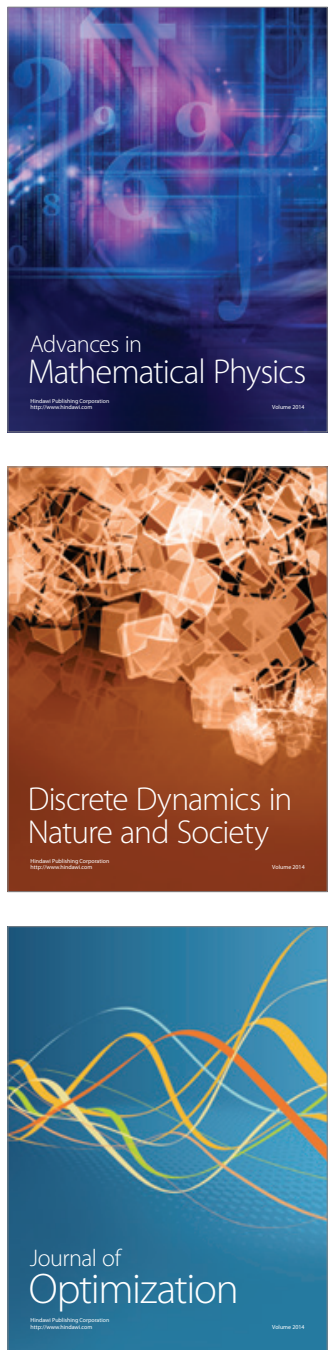